\title{
The Development of a Virtual Reality Environment to Model the Experience of Schizophrenia
}

\author{
Jennifer Tichon ${ }^{1}$, Jasmine Banks ${ }^{2}$, and Peter Yellowlees ${ }^{1}$ \\ 1 Centre for Online Health, University of Queensland, \\ Brisbane 4072, Australia \\ jtichon@ccs.uq.edu.au \\ p.yellowlees@mailbox.uq.edu.au \\ http://www.coh.uq.edu.au/ \\ 2 Advanced Computational Modelling Centre, University of Queensland, \\ Brisbane 4072, Australia \\ jbanks@maths .uq. edu . au \\ http://www.acmc.uq.edu.au/
}

\begin{abstract}
Virtual Reality (VR) techniques are increasingly being used for education about and in the treatment of certain types of mental illness. Research indicates that VR is delivering on its promised potential to provide enhanced training and treatment outcomes through incorporation of this high-end technology. Schizophrenia is a mental disorder affecting $1-2 \%$ of the population, and it is estimated $12-16 \%$ of hospital beds in Australia are occupied by patients with psychosis. Tragically, there is also an increased risk of suicide associated with this diagnosis. A significant research project being undertaken across the University of Queensland faculties of Health Sciences and EPSA (Engineering, Physical Sciences and Architecture) has constructed a number of virtual environments that reproduce the phenomena experienced by patients who have psychosis. Symptoms of psychosis include delusions, hallucinations and thought disorder. The VR environment will allow behavioral, exposure therapies to be conducted with exactly controlled exposure stimuli and an expected reduction in risk of harm. This paper reports on the current work of the project, previous stages of software development and the final goal to introduce VR to medical consulting rooms.
\end{abstract}

\section{Introduction}

Schizophrenia is a debilitating mental illness that affects $1-2 \%$ of the population at some point in their lives. It is estimated $12-16 \%$ of hospital beds in Australia are taken up with patients with schizophrenia. Tragically, suicide is more common among sufferers of this illness, which often strikes people in their prime. Psychotic symptoms associated with schizophrenia include delusions, hallucinations, and thought disorder [1]. Most people with psychotic symptoms "hear" voices, and a large proportion also "see" visual illusions. At present patients 
have to describe their hallucinations, auditory and visual, to their therapists there is no way that the therapists can either share the experiences or objectively evaluate them. As a consequence patients often feel that their therapists cannot really understand them, and the therapists themselves have difficulties learning about the exact nature of psychosis, as they have no personal experience of it.

The illusions experienced by patients with psychosis can be strange and often terrifying. Auditory hallucinations range in complexity from noises such as buzzes, bumps, screeches and muffled whispers, to ongoing multi- person discussions. The "voices" are often accusing or demeaning, and often giving paranoid suggestions to the patient. Auditory hallucinations include: audible thoughts that speak aloud what the patient is thinking; voices giving a running commentary on the patient's actions; two or more persons conversing, often about the patient who is referred to in the third person; and commands ordering the patient to do things. However they can also be frightening or dangerous, commanding acts of violence toward self or others.

Visual illusions range from simple flashes of light, subtle changes to objects, to elaborate visions. Visual hallucinations are usually seen nearby, clearly defined, in color, life-size, 3-dimensional, and moving. Subtle illusions include straight lines appearing curved, shimmering, slight movements, and objects appearing emphasized. People in pictures, on TV and in real life may appear to undergo subtle facial changes, or even to some extent morph into other entities, making their identity doubtful to the patient and increasing the patient's paranoia. Visual illusions will usually be accompanied by audio hallucinations, for example, pictures of people may morph into other entities and "talk" to the patient.

Virtual reality (VR) provides a real option for translating a person's psychological experience into a real experience others can share through the development of a virtual world that replicates the patient's world. VR techniques are increasingly being used in trial clinical programs and in the treatment of certain types of mental illness. The phenomenon of users of VR becoming immersed in virtual environments provides a potentially powerful tool for mental health professionals [23]. VR has been shown to help improve patient care in the form of advanced therapeutic options [4,5,6]. The research team at the University of Queensland have been collaborating for the past twelve months in the development of a VR tool to use in education, training and treatment of schizophrenia.

\section{The VISAC Laboratory and Remote Visualisation}

The Visualisation and Advanced Computing Laboratory (VISAC) at the University of Queensland consists of an immersive curved screen environment of $2.5 \mathrm{~m}$ radius and providing 150 degrees field of view. Three projectors separated by 50 degrees are used to project the images onto the curved screen. The curved screen environment is suitable for having small groups of people, eg patients and caregivers, to share the immersive experience.

The high quality infrastructure available at the University of Queensland provides a unique opportunity in Australia to undertake this collaborative project. 
The university arguably has the most sophisticated advanced computing infrastructure in Australia. Within the last nine months it has established a two million dollar state-of-the-art virtual reality centre, which is linked to a 40 million dollar supercomputer and a 20 terabyte mass storage facility. These facilities are managed by the Advanced Computational Modelling Centre (ACMC). The Centre has around 20 academic staff with expertise in scientific modelling, advanced computing, visualisation and bioinformatics (see http://www.acmc.uq.edu.au).

Through the Queensland Parallel Supercomputing Foundation (QPSF), the University of Queensland is a participant in the recently funded GrangeNet. GrangeNet will provide an upgrade in bandwidth between Sydney, Melbourne, Canberra and Brisbane to approximately $2.5 \mathrm{~GB} / \mathrm{sec}$. This will allow us to trial remote and collaborative visualisation between separate international locations.

Another aspect of this participation in GrangeNet is that the University of Queensland will build an area node within the next few months. This will enable us to perform multicasting between various international, national and regional sites with this facility and be able to display the visual images generated within VISAC around the world.

\section{Aims of the Project}

Using facilities of the VISAC laboratory, the project aims to artificially model the experience of psychosis in virtual environments, focusing on re-creating the audio hallucinations and visual illusions.

The ultimate goal of the project is to develop software that patients can use, in conjunction with their therapist, to re-create their experiences, in order that their therapists can better understand their symptoms, and allow for enhanced monitoring of their illness. It is envisaged that this environment could also be used as a structured and repeatable trigger for neurophysiological examinations of the central nervous system, and it may assist in understanding the intracerebral pathways of psychosis, and unraveling the mysteries of illnesses such as schizophrenia and psychosis in general.

To ensure that the re-created virtual world accurately models the patients' inner world, actual patients with schizophrenia have been interviewed and asked to describe their symptoms in detail. These descriptions have then been transcribed into models of the hallucinations that are personal to individual patients. What is being created are realistic environments where patients can be immersed into a virtual world created to specifically reflect the illusions for which they require treatment. Patients are interviewed for both feedback and evaluation on their individualised VR programme.

The next phase of the research project is to test its clinical potential. Case studies will be conducted where patients will be exposed to the specific hallucinations they have described and have been developed in the visualisation laboratory for them. The patient will be exposed to these hallucinations during therapy sessions. Measures of pre- and post-treatment on depression and symp- 
toms of schizophrenia will be made to ascertain whether VR was an effective intervention tool.

In attempting to develop a realistic, virtual environment which can simulate a psychosis such as schizophrenia, the project also aims to provide remote display of the visual models over a high speed communications network. Therefore, web enabling of visual models for efficient and effective on-line access is also being examined.

\section{Software Development}

The project commenced in October 2001, and since then, has undergone a number of distinct development phases.

Phase 1. The initial work involved building a model of an everyday environment, in this case a living room, using a commercial 3D modelling package. This room contained standard furniture including a sofa, coffee table, dining table, chairs and bookshelf, as well as a number of objects including a TV, radio, pictures and painting, which were believed to trigger hallucinations. The hallucinations modelled included: a face in a portrait morphing from one person into another and also changing its facial expression; a picture on the wall distorting; the walls of the room contracting and distorting so that the straight edges of the walls appeared curved; the blades of a ceiling fan dipping down; and the TV switching on and off of its own accord. In addition, a soundtrack of auditory hallucinations, provided by the pharmaceutical company Janssen-Cilag, was played in the background as these visual hallucinations were taking place. This was in order to give a good approximation to the cacophony of voices that would be going on whilst a patient is trying to concentrate on everyday tasks.

Two screenshots of the living room model are shown in Fig. 1] Figure 2] shows the painting on the wall, before and after the distortion process.

This initial concept of modelling a set of psychotic experiences was shown to a number of patients. Feedback from patients and mental health care professionals
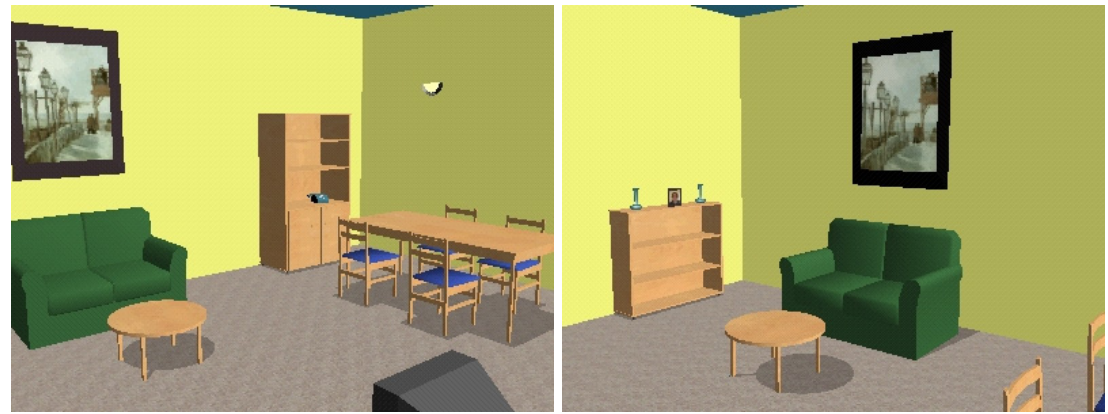

Fig. 1. Screen shots of the living room model 


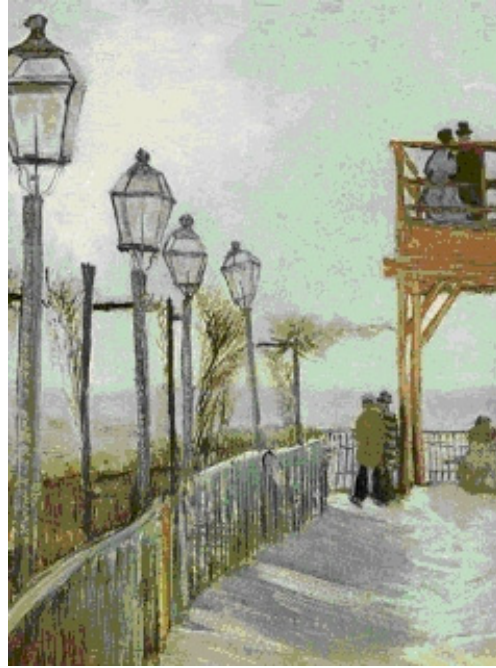

a

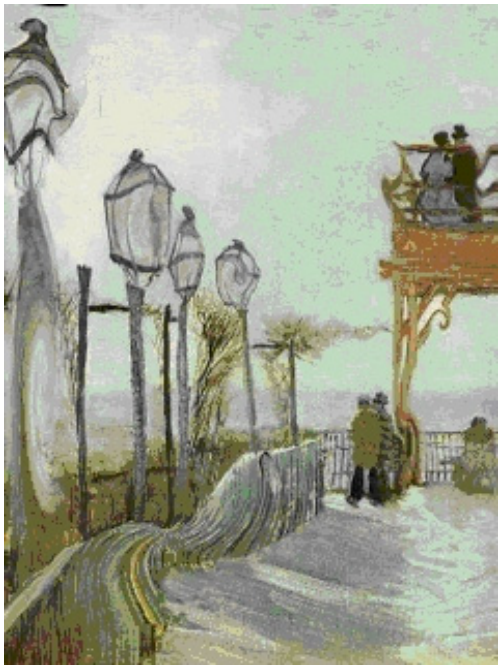

b

Fig. 2. Wall painting from the living room scene $\mathbf{a}$ original $\mathbf{b}$ distorted

was generally positive. However, due to the generic nature of the auditory and visual hallucinations portrayed, it was not possible to confirm how realistic the model was. A number of patients commented that they liked the idea, however, felt that the hallucinations modelled did not actually relate to them.

Phase 2. The second phase of the project involved modelling the experiences of one particular patient who agreed to be involved in the project. The models of the hallucinations were then implemented based on the patient's descriptions. In this way, a model of psychosis would be built from the patient's perspective.

The first challenge involved selecting a suitable environment in which the hallucinations could be modelled. A psychiatric ward was chosen, as it is an environment in which many patients would have experienced hallucinations, and could therefore be used as a basis for modelling the hallucinations of subsequent patients who become involved in the project. It also contains a variety of different rooms such as bedrooms, bathrooms, a common room, offices and hallways where hallucinations may occur.

Development of the virtual environment for Phase 2 comprised two main steps. The first step involved creating the model of the psychiatric ward, and models of static elements of the scene (eg. furniture) using a 3D modelling package. In order to build a model of a psychiatric ward and the objects within it, we visited the psychiatric unit at the Royal Brisbane Hospital, and took photographs of various textures and room layouts. This enabled the final model to be as realistic as possible. The static models of the psychiatric ward and objects were saved as VRML files for inclusion into the main program. Some views of the virtual psychiatric ward are shown in Fig. 3. 


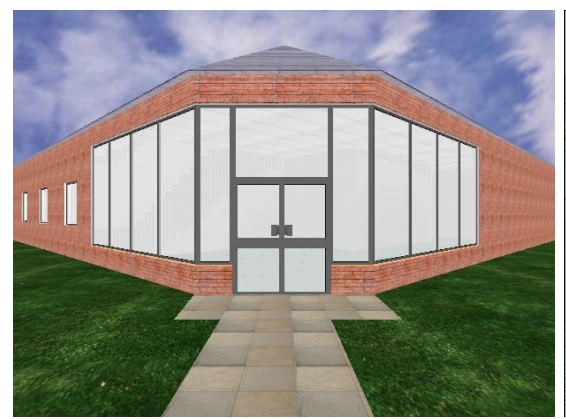

a

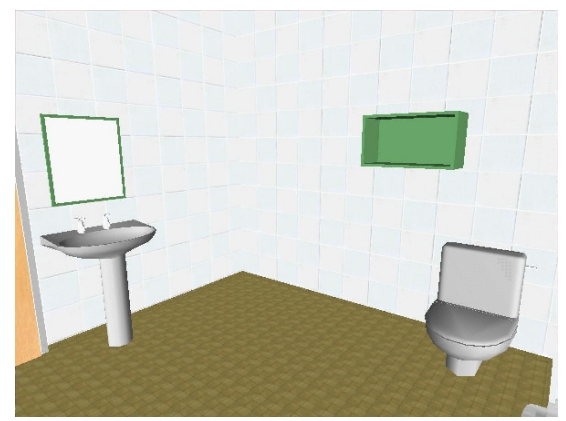

C

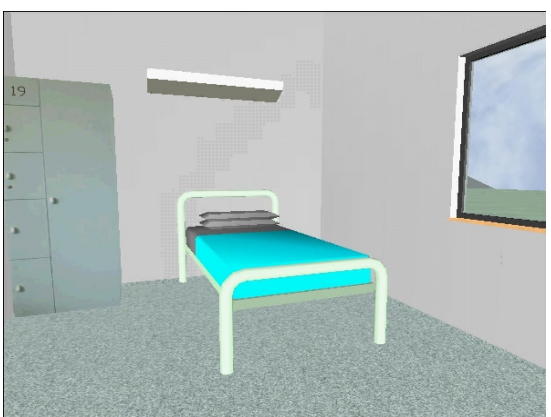

b

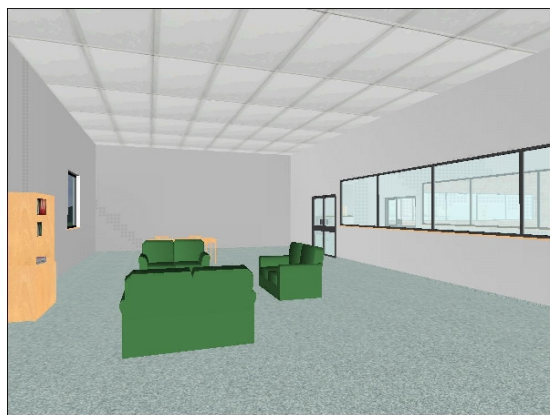

d

Fig. 3. Views of the psychiatric ward model $\mathbf{a}$ front $\mathbf{b}$ bedroom $\mathbf{c}$ bathroom $\mathbf{d}$ common room

The second stage of development involved writing the main program which loads, positions and displays the static elements, and also which implements the dynamic parts of the scene, such as sounds and movements of objects. The software was written in $\mathrm{C} / \mathrm{C}++$, in conjunction with an open source, cross platform scene graph technology. This method of implementation was chosen as it will allow us to eventually port the software from the current IRIX platform to a PC platform. This would enable the software to be used, for example, in a psychiatrist's office or in a hospital, making it more accessible to patients, caregivers and mental health workers.

The software was designed so that the user is able to navigate around the scene using the mouse and keyboard, and various hallucinations are triggered either by proximity to objects, by pressing hot keys, or by clicking an object with the mouse.

One particularly challenging hallucination modelled was a vision of the Virgin Mary, which would appear and "talk" to the patient. At first, it was thought that this would be implemented as a 3D model, however, this would have introduced a great deal more polygons and complexity in to the scene and slowed down performance. It was decided that a $2 \mathrm{D}$ film projected onto a billboard would give adequate performance and create an apparition- like effect. Therefore, the 
Virgin Mary sequence was filmed using an actor, and the background set to transparent, so that only the apparition could be seen against the background of the psychiatric ward. A separate program was written to preprocess the movie, to remove some artifacts and to add a golden halo around the Virgin Mary figure. Other challenges with this hallucination involved making sure the movie and it's audio were in sync, and also achieving an adequate frame-rate.

Other hallucinations modelled include the word "Death" appearing to stand out of newspaper headlines, and random flashes of light. Some of these hallucinations are shown in Fig. 4 Audio hallucinations implemented included: a speech on the radio, which begins as a political speech, but changes to refer to the patient and concludes by commanding the patient to kill themselves; a chorus of music playing; voices discussing the patient in a derogatory manner; and other sounds and voices telling the patient they are evil; and laughter. The audio hallucinations can be triggered by proximity to a certain object (such as the stereo), or they can also play continuously in the background at random intervals as one "walks" through the virtual scene. In this way, it gives the effect of many different sounds and voices interjecting and happening simultaneously. It is also quite repetitive with the sounds and voices playing over and over again.

\section{Feedback}

The patient was positive about the simulation, stating "It's just such an extraordinary experience" and "It was a quite good approximation capturing the essence of the experience." The patient particularly liked how the visions and voices interlinked and were played over one another. This effect reportedly effectively re-created "the sensation of a lot going on in your head." Also, they liked the way that the hallucinations repeated themselves. "The fact that they went on and on and people would think, 'My god, why can't they turn off.' They go on and on - and how you just can't step out of the room and get away from them."

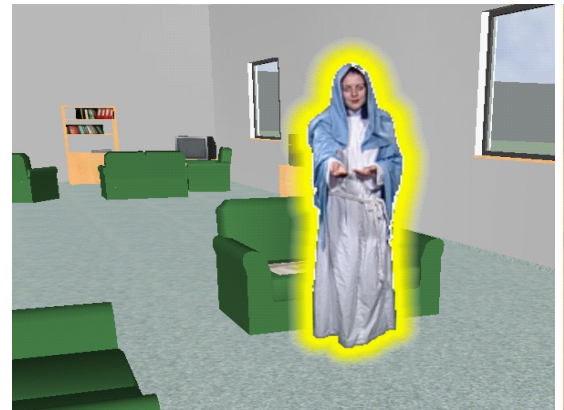

a

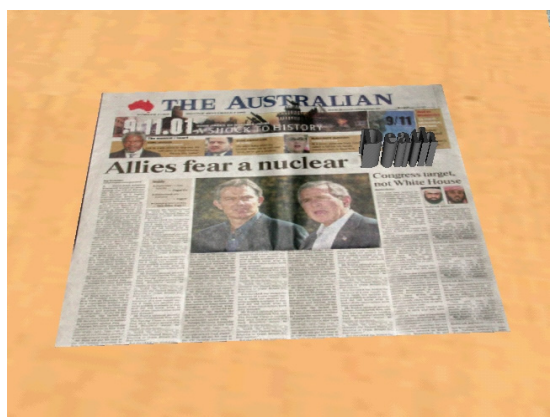

b

Fig. 4. Hallucinations a Virgin Mary b "Death" headline 
The patient commented that the virtual environment was effective in recreating the same emotions that they experienced on a day-to-day basis during their psychotic episodes. This is perhaps the most important goal of the simulation - more important than scene realism - if the software is to be used for education and for increasing empathy with sufferers of psychosis.

\section{Further Work}

At present, a second patient has been interviewed, and the hallucinations of this patient are being modelled. The model of the psychiatric ward is being again used as the basis for this. Many of these hallucinations are quite different from those of the first patient and will require development of new techniques in order for them to be re-created. These include working out and efficient way to add human figures to the scene, implementing reflective surfaces, implementing fog effects, and adding more realistic lighting and shadow effects to the scene.

The software currently works only in the VISAC lab and on machines running IRIX, and will need to be ported to a PC platform such as Linux or Windows. This will mean that some parts of the code, such as the interface to the audio and movie libraries, will need to be re-written.

\section{Conclusion}

To date no research exploring the clinical use of VR in psychosis can be located. As outlined, the main aims of the project are the development of the virtual reality software for use in $3 \mathrm{D}$ environments and to design it to be deliverable on consulting room PCs. This project has the potential to have a significance impact on the field of psychiatry in both the assessment and in the on-going monitoring of patients with schizophrenia.

It is expected that the virtual environment will also provide an effective 3dimensional teaching and awareness environment, for mental health workers and students. It is known that a significant factor in the recovery of patients is the support they receive from family, caregivers and the community at large. It is hoped that this work will help family members of patients with schizophrenia to understand and empathise more with their loved one's experiences. Also, it is hoped that it will be used as an effective tool for improving the awareness and understanding of illnesses such as schizophrenia in the wider community, thus ultimately improving the quality of life and chances of recovery of patients.

Virtual Reality is changing the ways in which we learn about and treat medical conditions. Due to cost limitations the full potential of VR has not yet been realised. Projects such as this one are working to make state- of-the-art health technology more readily accessible and cost-effective to mental health practitioners. 


\section{References}

1. H. Kaplan and B. Sadock. Comprehensive Textbook of Psychiatry, volume 1\&2. Lippincott Williams Wilkin, Philadelphia, 7th edition edition, 2000.

2. L. Hodges, P. Anderson, G. Burdea, H. Hoffman, and B. Rothbaum. Treating psychological and physical disorders with VR. IEEE Computer Graphics and Applications, pages 25-33, Nov-Dec 2001.

3. M. Kahan. Integration of psychodynamic and cognitive-behavioral therapy in a virtual environment. Cyberpsychology and Behavior, 3:179-183, 2000.

4. G. Riva and L. Gamberini. Virtual reality in telemedicine. Cyberpsychology and Behavior, 6:327-340, 2000.

5. G. Riva. From telehealth to e-health: Internet and distributed virtual reality in health care. Cyberpsychology an Behavior, 3:989-998, 2000.

6. P. Anderson, B. Rothbaum, and L. Hodges. Virtual reality: Using the virtual world to improve quality of life in the real world. Bulletin Menninger Clinic, 65:78-91, 2001. 